http://jmscr.igmpublication.org/home/

ISSN (e)-2347-176x ISSN (p) 2455-0450

crossref DOI: https://dx.doi.org/10.18535/jmscr/v7i8.96

Journal Of Medical Science And Clinical Research

$\underline{\text { Research Article }}$

\title{
Role of Boerhaavia Diffusa L: A In Relief of Neuropathic Pain of Paclitaxel Induced Neuropathy
}

\author{
Authors \\ Dr Sandeep Kamlekar ${ }^{*}$, Dr Jamila Tahashildar ${ }^{2}$, Dr Sangita Gupta ${ }^{3}$ \\ ${ }^{1}$ Assistant Professor, ${ }^{2}$ Professor, ${ }^{3}$ Professor \& HOD \\ Department of pharmacology, Geetanjali Medical College \& Hospital, Udaipur, Rajasthan \\ *Corresponding Author \\ Dr Sandeep Kamlekar
}

Assistant Professor; Department of Pharmacology, Geetanjali Medical College \& Hospital, Udaipur,

Rajasthan, India

\begin{abstract}
Background: The present study was designed to investigate the neuropathic activity of Boerhaavia diffusa L. against Paclitaxel induced peripheral neuropathy in rats.

Materials and Methods: Peripheral neuropathy was induced by a single i.p injection of Paclitaxel $2 \mathrm{mg} / \mathrm{kg}$ for four alternative days 0, 2,4,6. The ethanolic extract of Boerhaavia diffusa was administered 100, 200, $400 \mathrm{mg} / \mathrm{kg}$, p.o. daily up to 28 days. Neuropathic pain was assessed via various behavioral parameters procedures viz., Eddy's hot plate test and cold water tail immersion test, pinprick, Acetone drop, photoactometer tests were performed to assess the degree of thermal, mechanical hyperalgesia, cold allodynia along with locomotor activity.

Result: In this study Paclitaxel induced rats showed decreased tail withdrawal latency, and increased paw withdrawal latency along with decreased locomotor activity was observed for various behavioural tests. After treating rats with Boerhaavia diffusa L. at various doses 100, 200, $400 \mathrm{mg} / \mathrm{kg}$, p.o. daily up to 28 days, the animals showed improvement in tail and paw withdrawal latency and locomotor activity in dose dependent manner.

Conclusion: The present study reveals the supplementation of B. diffusa L., extract ameliorates the pacliatxel induced neuropathy via above mentioned actions.

Keywords: Neuropathic pain, Paclitaxel, Boerhaavia diffusa L.
\end{abstract}

\section{Introduction}

Neuropathic pain (NP) is a manifestation of multiple and varied disorders that display both peripheral and central sensitization mechanisms. ${ }^{1}$ It affects somatosensory components of the nervous system. ${ }^{2}$ A prolonged sensory input from injured peripheral nerve generate secondary morphological changes in dorsal regulatory ganglion (DRG), and formation of new ion channels and receptors at the cellular level result in hyper excitability of neurons. ${ }^{3}$ In fact, many pathological conditions like cancer, AIDS, long standing diabetes, lumbar disc syndrome, herpes infection, multiple sclerosis and stroke causative for peripheral nerve damage and pain. ${ }^{4}$ 
The prevalence of cancer is increasing globally; around 17 million new cases were estimated by the year 2020., Approximately, 40\% cancer patients are sufferers of peripheral neuropathy during chemotherapy treatment with vincristine, paclitaxel (Taxol), platinum compounds. ${ }^{7}$ The neuropathy inducing potential of these drugs depends on their cumulative dose, time, and mechanism of binding. ${ }^{8}$

Paclitaxel induced peripheral neuropathy occur due to microtubule dysfunction in dorsal root ganglia, axons and schwann cells. ${ }^{9}$ The neuropathy will begin as early as in 24 to 72 hours after administering single, high dose of Paclitaxel. ${ }^{10}$ The generating symptoms starts from lower extremities like pins and needles, burning, decreased or increased sensitivity to pain endanger quality of life. ${ }^{11}$ The type of pain and generating symptoms are poorly treated with available drugs. 12

Preclinically, various studies have been reported on plant products like aconittuber, lindera angustifolia, teucrium polium, phyllanthus emblica, vochysia divergens, cannabis sativa, nigella sativa, ocimum sanctum and ginkgo biloba in management of neuropathy. ${ }^{13}$ Therefore ample of scope for developing the novel medicinal agents from the plant origin for treating neuropathic pain.

The present chosen trailing herb bearing a Latin name; Boerhaavia diffusa Linn. (Family: Nyctaginaceae) is a creeping herb grown in tropical regions of South America, India and Africa. Indian Ayurveda renowned it as 'Punarnava', rejuvenates itself from dried root in rainy seasons as well as rejuvenates body. The whole plant has medical benefits especially roots. There, the roots are employed for multiple purposes including liver, gallbladder, and kidney, renal and urinary disorders. It is a very good nerve rejuvenator and it is given in cases of sciatica or nervous weakness or even paralysis conditions to relive pain. Traditionally Punarnava root used as anticonvulsant, analgesic, expectorant, CNS depressant, laxative, diuretic, abortifaci and swelling. ${ }^{14}$ The plant is rich in photochemicals like phytochemicals like Punarnavine (alkaloid), boeravinone (rotenoid), flavonoids, amino acids, liriodendrons (Lignans), B-sistosterol and stetracosanoic acid, ecosanoic, steroic and urosolic acid has proven to cure and control disease prognosis. ${ }^{15}$

Thus, the present study is aimed to investigate the neuropathic pain relieving activity, antioxidant activity of Boerhaavia diffusa L., against the Paclitaxel induced neuropathy in albino rats.

\section{Materials and Methods}

\section{Chemicals and reagents}

Paclitaxel was obtained from Hetero Health care LTD, Hyderabad, India and pregabalin was purchased from Swapnaroop drugs \& pharmaceuticals, Aurangabad, Maharashtra, India. All other chemical were used are analytical grade.

Plant material collection and extraction: The plant Boerhaavia diffusa L., was gathered from local fields in the month of October-November, it was authenticated with a botanist. The roots were separated and thoroughly cleaned with distilled water, and shade dried until they were free from moisture. The dried roots were subjected to size reduction with a mechanical grinder until fine coarse powdered granules would obtain. The obtained granular powder was undergone cold maceration in $95 \%$ ethanol for a period of $72 \mathrm{hrs}$ with frequent agitation in a closed neck glass bottle. The obtained visibly green coloured liquid containing soluble phytochemical constituent's undergone filtration through whatman \# 1 filter paper and further concentrated at $45^{\circ} \mathrm{C}$ on a water bath to obtain a sticky brownish semisolid paste. 16 The extracted semisolid paste was undergone drying in desiccators to remove moist and stored at below $10^{0} \mathrm{C}$. The obtained yield in \% (2.54 gm w/w) was calculated with reference to the powder used for extraction.

According to OECD 420 guidelines study was conducted on healthy albino rats (150-200 g), sub maximal and supra maximal doses of $200 \mathrm{mg} / \mathrm{kg}$ were selected. ${ }^{17}$ 
Animal selection and induction of Neuropathy

After taking approval from Institutional Animal Ethical Committee (IAECH) healthy adult albino rats of either sex 150-200 gm were selected for the study. The animals were housed in clean, transparent polypropylene cages with six animals in each cage by maintaining standard laboratory conditions 12:12 hrs light and dark cycles, at $25 \pm 3^{0} \mathrm{C}$ and $35-60 \%$ humidity and had free accesses to water with standard chow diet. The study was approved by Institutional Animal Ethical Committee (IAEC).

\section{Treatment Protocol}

Six experimental groups were assigned as Control, Disease control, Standard, and three groups of test drugs at various doses, expressed as $0.9 \%$ N.S (p.o), Paclitaxel (i.p.) $2 \mathrm{mg} / \mathrm{kg}$, four alternative days $0,2,4,6$ in early morning as described previously. ${ }^{18}$ Pregabalin $10 \mathrm{mg} / \mathrm{kg}$ (p.o), B. Diffusa L. 100, 200, $400 \mathrm{mg} / \mathrm{kg}$ (p.o) by dissolving in distil water respectively 1 hour prior commencing the experiment. On $7^{\text {th }}$ day the animals diagnosed neuropathic pain in 5 groups. From $7^{\text {th }}$ day animals fed with Pregabalin and B.diffusa L., respectively one hour before doing the behavioural parameter tests. The pain threshold was assessed by cold water tail immersion test and paw heat hyperalgesia, acetone drop test, pinprick test, and actophotmeter test, were used to assess the Behavioural alterations according the standard methods ${ }^{18}$. The assessments were done on day 0 (before administering Paclitaxel) and after on days 7, 14, 21, 28 respectively. At the end of the study (i.e., $28^{\text {th }}$ day) rats was sacrificed by administering thiopental-50 mg/kg, IP, and a $10 \% \mathrm{w} / \mathrm{v}$ nerve homogenate was prepared by mixing Triss-Hcl buffer $\left(\mathrm{P}^{\mathrm{H}} 7.4\right)$. The nerve homogenate was further cooled in ice cold water at $4^{0} \mathrm{C}$ and centrifuged at $2500 \mathrm{rpm}$ for $10 \mathrm{~min}$. The obtained supernatant was subjected to estimate for total protein, total calcium, lipid peroxidation, superoxide dismutase levels ${ }^{18}$ was estimated.

\section{Statistical Analysis}

The results were expressed as mean $\pm \mathrm{SD}$. The data was entered in Microsoft excel and analysis was done by using multiple comparison tests by using ANOVA followed by Bonferroni t-test. The statistical analysis was done by using the Sigma Stat 3.5.values of $\mathrm{P}<0.05$ were considered statistically significant.

\section{Results \\ Behavioural Parameters \\ Cold water tail immersion test}

This group also showed similar results with B. diffusa L. $100 \mathrm{mg} / \mathrm{kg}(7.833 \pm 0.752,8.83 \pm 0.983$, $9.16 \pm 0.983,9.83 \pm 0.983), 200 \mathrm{mg} / \mathrm{kg}(8.50 \pm 1.048$, $9.66 \pm 632,10.50 \pm 1.516,10.83 \pm 1.471), 400 \mathrm{mg} / \mathrm{kg}$ p.o $(10.16 \pm 1.471, \quad 10.16 \pm 1.505, \quad 12.00 \pm 1.264$, $12.50 \pm 1.048)$ were shown significant $(\mathrm{p}<0.001)$ value as above group in comparison with disease control group from day 7 to 28 days. Whereas tail withdrawal latency of Paclitaxel induced rats was decreased in comparison with normal rats from day 7 to 28 day $(10.16 \pm 0.752,10.66 \pm 0.816$, $10.83 \pm 0.752,11.00 \pm 1.264)$. [Table-I]

Eddy's hot plate test: Paclitaxel treated rats showed decreased paw withdrawal latency in comparison with normal rats from day 7 to 28 days $\quad(8.33 \pm 0.816, \quad 7.16 \pm 0.752, \quad 6.00 \pm 0.894$, $5.00 \pm 0.632$ ). The higher dose of B. diffusa L., 400 $\mathrm{mg} / \mathrm{kg}$ only given early significant $(\mathrm{P}<0.01)$ response from day $7(11.16 \pm 1.169,12.50 \pm 1.048$, $12.16 \pm 0.752,14.00 \pm 0.632$ ) in comparison with and remaining two doses 100, $200 \mathrm{mg} / \mathrm{kg}$ were unsuccessful. [Table-II]

Pinprick Test: Rats treated with Paclitaxel causative for development of mechanical hyperalgesia observed as increased paw withdrawal latency from a nicking object in comparison with normal rats from day 7 to 28 $(10.33 \pm 0.816, \quad 12.50 \pm 0.547, \quad 14.16 \pm 0.752$, $15.33 \pm 0.816)$. The dose $400 \mathrm{mg} / \mathrm{kg}$ of B. diffusa were shown a significant $(\mathrm{P}<0.001)$ early response in decreased in paw withdrawal latency from day $7 \quad(7.33 \pm 1.36, \quad 8.66 \pm 1.211, \quad 10.16 \pm 1.16$, $11.5 \pm 1.048)$ and dose $200 \mathrm{mg} / \mathrm{kg}$ were given late 
significant response $(\mathrm{P}<0.05)$ from day 14 $(10.33 \pm 1.211, \quad 11.50 \pm 1.048, \quad 12.66 \pm 1.032) \quad$ in comparison with Paclitaxel. [Table-III]

Acetone drop test: The acetone increases paw withdrawal latency in Paclitaxel treated rats $(14.06 \pm 1.063, \quad 23.72 \pm 2.560, \quad 32.89 \pm 1.834$, $43.06 \pm 2.323$ ) in comparison with normal rats from day 7 to 28 . All three doses of B. diffusa were successful in decreasing paw withdrawal latency. The test dose $400(9.00 \pm 0.843,13.94 \pm 1.597$, $16.78 \pm 2.810, \quad 17.94 \pm 2.776)$ and $200 \mathrm{mg} / \mathrm{kg}$ $(10.44 \pm 1.004, \quad 17.22 \pm 1.109, \quad 21.28 \pm 2.081$, $27.33 \pm 2.271$ ) of $B$. diffusa were shown early significant results on day $7(\mathrm{P}<0.001)$ and dose $100 \mathrm{mg} / \mathrm{kg} \quad(19.67 \pm 2.044, \quad 26.83 \pm 2.834$, $35.72 \pm 3.172)$ was on day $14(\mathrm{P}<0.01)$. [Table-IV] Locomotor Activity Test: Paclitaxel treated rats showed decreased locomotor activity in comparison with normal rats from day 7 to 28 $(42.67 \pm 7.033, \quad 38.83 \pm 6.494, \quad 35.67 \pm 6.154$, $39.33 \pm 7.005)$. All the tree doses of B. diffusa were successful in improving locomotor activity in comparison with diseased control rats. The early significant effect was observed with $B$. diffusa dose $100 \mathrm{mg} / \mathrm{kg}$ dose on $21 \mathrm{st}$ day $(52.33 \pm 5.086$, $55.83 \pm 4.535) ; \quad 200 \quad \mathrm{mg} / \mathrm{kg} \quad(56.17 \pm 6.616$, $64.67 \pm 6.772,69.67 \pm 6.218)$ on day 14 , and with $400 \mathrm{mg} / \mathrm{kg}$ day $7(54.33 \pm 5.538,63.83 \pm 6.464$, $71.50 \pm 7.007, \quad 78.33 \pm 7.554) \quad$ significantly (P<0.001). [Table-V]

In all behavioural parameters pregabalin showed early significant results $(\mathrm{P}<0.001)$ from day 7 to 28 days.
(Cold water tail immersion: 12.16 \pm 0.983 , $12.16 \pm 0.983,12.83 \pm 1.471,13.16 \pm 1.329$; Eddy's hot plate: $11.66 \pm 0.816, \quad 13.00 \pm 0.632,14.16 \pm$ $0.408,15.16 \pm 0.752$; Pin prick test: $6.00 \pm 1.095$, $7.33 \pm 1.211,8.66 \pm 1.032,9.50 \pm 1.048$; Acetone drop test: $6.56 \pm 0.544,8.11 \pm 1.109,8.61 \pm 1.163$, $7.89 \pm 1.004$; Locomotor activity test: $60.67 \pm 4.227$, $74.17 \pm 5.345,83.50 \pm 5.753,91.17 \pm 5.947)$.

\section{Biochemical Parameters}

Effect of B. diffusa on Lipid peroxidation levels (TBARS), total protein, total calcium, superoxide desmutase levels ( $\mathrm{nM} / \mathrm{gm}$ of wet tissue) in sciatic nerve

In 28days of study Paclitaxel treated rats were shown increased levels of lipid per oxidation (TBARS) levels $(54.55 \pm 10.296)$, calcium levels $(26.7 \pm 2.654)$ and decreased levels of total protein $(34.16 \pm 4.633)$ and superoxide dismutase levels $(13.15 \pm 6.696)$ was noted significantly $(\mathrm{P}<0.05)$ in comparison with normal control rats. The test dose $400 \mathrm{mg} / \mathrm{kg}$ of B. diffusa reduces the raised lipid peroxidation (TBARS) levels $(36.50 \pm 6.992)$ and improves total protein levels $(53.91 \pm 3.679)$ significantly $(\mathrm{P}<0.001)$; while the superoxide dismutase levels were more successfully raised by $400 \quad \mathrm{mg} / \mathrm{kg} \quad(28.05 \pm 6.105), \quad 200 \quad \mathrm{mg} / \mathrm{kg}$ $(23.33 \pm 4.190)$ test doses significantly $(\mathrm{P}<0.001)$ in Paclitaxel induced rats. The raised calcium levels due to Paclitaxel therapy were successfully blocked by $200 \quad(21.20 \pm 1.930), 400 \mathrm{mg} / \mathrm{kg}$ $(18.18 \pm 1.772)$ doses of $\mathrm{B}$. diffusa significantly $(\mathrm{P}<0.001)$. [Table-VI]

Table-I: Effects of B. diffusa L., on cold water tail immersion test in paclitaxel induced rats

\begin{tabular}{|l|c|c|c|c|c|}
\hline \multirow{2}{*}{ Treatment groups } & \multicolumn{5}{|c|}{ Reaction time (Sec) (n=6) } \\
\cline { 2 - 6 } & Day-0 & Day-7 & Day-14 & Day-21 & Day-28 \\
\hline Normal Control & $11.5 \pm 1.04$ & $10.16 \pm 0.752$ & $10.66 \pm 0.816$ & $10.83 \pm 0.752$ & $11.00 \pm 1.264$ \\
\hline Paclitaxel (Disease control) & $11.33 \pm 1.03$ & $5.166 \pm 0.752$ & $4.50 \pm 1.048$ & $3.833 \pm 0.752$ & $3.50 \pm 0.547$ \\
\hline $\begin{array}{l}\text { Paclitaxel + Pregabalin } \\
(10 \mathrm{mg} / \mathrm{kg})\end{array}$ & $11.41 \pm 1.42$ & $12.16 \pm 0.983$ & $12.16 \pm 0.983$ & $12.83 \pm 1.471$ & $13.16 \pm 1.329$ \\
\hline $\begin{array}{l}\text { Paclitaxal + } \\
\text { B. diffusa (100 mg/kg) }\end{array}$ & $11 \pm 1.41$ & $\begin{array}{c}7.833 \pm 0.752 \\
* *\end{array}$ & $\begin{array}{c}8.83 \pm 0.983 \\
* * *\end{array}$ & $\begin{array}{c}9.16 \pm 0.983 \\
* * *\end{array}$ & $\begin{array}{c}9.83 \pm 0.983 \\
* * *\end{array}$ \\
\hline $\begin{array}{l}\text { Paclitaxel }+ \\
\text { B. diffusa }(200 \mathrm{mg} / \mathrm{kg})\end{array}$ & $11.33 \pm 0.81$ & $\begin{array}{c}8.50 \pm 1.048 \\
\text { Paclitaxel }+\end{array}$ & $\begin{array}{c}9.66 \pm 1.632 \\
* * *\end{array}$ & $\begin{array}{c}10.50 \pm 1.516 \\
* * *\end{array}$ & $\begin{array}{c}10.83 \pm 1.471 \\
* * *\end{array}$ \\
\hline B. diffusa $(400 \mathrm{mg} / \mathrm{kg})$ & $11.83 \pm 1.47$ & $\begin{array}{c}10.16 \pm 1.471 \\
* * *\end{array}$ & $\begin{array}{c}11.33 \pm 1.505 \\
* * *\end{array}$ & $\begin{array}{c}12.00 \pm 1.264 \\
* * *\end{array}$ & $\begin{array}{c}12.50 \pm 1.048 \\
* * *\end{array}$ \\
\hline
\end{tabular}


Table-II: Effects of B. diffusa L., on thermal hyperalgesia by Eddy's hot plate test in paclitaxel induced rats

\begin{tabular}{|l|c|c|c|c|c|}
\hline \multirow{2}{*}{ Treatment groups } & \multicolumn{5}{|c|}{ Reaction time (Sec) (n=6) } \\
\cline { 2 - 6 } & Day-0 & Day-7 & Day-14 & Day-21 & Day-28 \\
\hline Normal control & $11.83 \pm 1.16$ & $11.66 \pm 1.032$ & $12.16 \pm 0.752$ & $12.66 \pm 1.032$ & $12.33 \pm 1.032$ \\
\hline Paclitaxel (Disease control) & $12 \pm 1.26$ & $8.33 \pm 0.816$ & $7.16 \pm 0.752$ & $6.00 \pm 0.894$ & $5.00 \pm 0.632$ \\
\hline $\begin{array}{l}\text { Paclitaxel + Pregabalin } \\
(10 \mathrm{mg} / \mathrm{kg})\end{array}$ & $12 \pm 1.26$ & $11.66 \pm 0.816$ & $13.00 \pm 0.632$ & $14.16 \pm 0.408$ & $15.16 \pm 0.752$ \\
\hline $\begin{array}{l}\text { Paclitaxel + } \\
\text { B. diffusa (100 mg/kg) }\end{array}$ & $12.16 \pm 1.47$ & $9.66 \pm 1.211$ & $10.833 \pm 0.752$ & $11.66 \pm 0.516$ & $12.16 \pm 0.752$ \\
\hline $\begin{array}{l}\text { Paclitaxel + } \\
\text { B. diffusa (200 mg/kg) }\end{array}$ & $12.33 \pm 0.81$ & $10.50 \pm 1.378$ & $11.83 \pm 1.169$ & $12.83 \pm 0.983$ & $13.33 \pm 0.816$ \\
\hline $\begin{array}{l}\text { Paclitaxel + } \\
\text { B. diffusa }(400 \mathrm{mg} / \mathrm{kg})\end{array}$ & $11.83 \pm 1.47$ & $\begin{array}{c}11.16 \pm 1.169 \\
* *\end{array}$ & $\begin{array}{c}12.50 \pm 1.048 \\
* * *\end{array}$ & $\begin{array}{c}13.16 \pm 0.752 \\
* * *\end{array}$ & $\begin{array}{c}14.00 \pm 0.632 \\
* * * *\end{array}$ \\
\hline
\end{tabular}

$* \mathrm{P}<0.05, * * \mathrm{P}<0.01, * * * \mathrm{P}<0.001$ compared with disease group.

Table-III: Effects of B. diffusa L., on prick induced hyperalgesia by pin prick test in paclitaxel induced rats:

\begin{tabular}{|l|c|c|c|c|c|}
\hline \multirow{2}{*}{ Treatment groups } & \multicolumn{5}{|c|}{ Reaction time (Sec) (n=6) } \\
\cline { 2 - 6 } & Day-0 & Day-7 & Day-14 & Day-21 & Day-28 \\
\hline Normal control & $3.16 \pm 0.75$ & $2.66 \pm 0.816$ & $2.83 \pm 0.752$ & $3.33 \pm 0.516$ & $3.50 \pm 0.547$ \\
\hline Paclitaxel (Disease control) & $3.5 \pm 0.83$ & $10.33 \pm 0.816$ & $12.50 \pm 0.547$ & $14.16 \pm 0.752$ & $15.33 \pm 0.816$ \\
\hline $\begin{array}{l}\text { Paclitaxel + Pregabalin } \\
(10 \mathrm{mg} / \mathrm{kg})\end{array}$ & $2.83 \pm 0.75$ & $6.00 \pm 1.095$ & $7.33 \pm 1.211$ & $8.66 \pm 1.032$ & $9.50 \pm 1.048$ \\
\hline $\begin{array}{l}\text { Paclitaxel + } \\
\text { B. diffusa (100 mg/kg) }\end{array}$ & $3.16 \pm 0.40$ & $9.66 \pm 1.505$ & $11.16 \pm 1.329$ & $12.66 \pm 1.032$ & $13.83 \pm 0.752$ \\
\hline $\begin{array}{l}\text { Paclitaxel + } \\
\text { B. diffusa (200 mg/kg) }\end{array}$ & $2.83 \pm 0.98$ & $9.00 \pm 0.894$ & $\begin{array}{c}10.33 \pm 1.211 \\
*\end{array}$ & $\begin{array}{c}11.50 \pm 1.048 \\
* * *\end{array}$ & $\begin{array}{c}12.66 \pm 1.032 \\
* * *\end{array}$ \\
\hline $\begin{array}{l}\text { Paclitaxel + } \\
\text { B. diffusa }(400 \mathrm{mg} / \mathrm{kg})\end{array}$ & $2.83 \pm 1.16$ & $\begin{array}{c}7.33 \pm 1.366 \\
* * *\end{array}$ & $\begin{array}{c}8.66 \pm 1.211 \\
* * *\end{array}$ & $\begin{array}{c}10.16 \pm 1.16 \\
* * *\end{array}$ & $\begin{array}{c}11.5 \pm 1.048 \\
* * *\end{array}$ \\
\hline
\end{tabular}

$* \mathrm{P}<0.05, * * \mathrm{P}<0.01, * * * \mathrm{P}<0.001$ compared with disease group.

Table-IV: Effects of B.diffusa L., on acetone drop test in paclitaxel induced rats

\begin{tabular}{|c|c|c|c|c|c|}
\hline \multirow[t]{2}{*}{ Treatment Groups } & \multicolumn{5}{|c|}{ Reaction time (Sec) $(n=6)$} \\
\hline & Day-0 & Day-7 & Day-14 & Day-21 & Day-28 \\
\hline Normal control & $6 \pm 0.596$ & $5.78 \pm 0.344$ & $5.94 \pm 0.491$ & $5.89 \pm 0.455$ & $5.67 \pm 0.298$ \\
\hline Paclitaxel (Disease control) & $6 \pm 0.471$ & $14.06 \pm 1.063$ & $23.72 \pm 2.560$ & $32.89 \pm 1.834$ & $43.06 \pm 2.323$ \\
\hline $\begin{array}{l}\text { Paclitaxel + Pregabalin } \\
(10 \mathrm{mg} / \mathrm{kg})\end{array}$ & $6 \pm 0.516$ & $6.56 \pm 0.544$ & $8.11 \pm 1.109$ & $8.61 \pm 1.163$ & $7.89 \pm 1.004$ \\
\hline $\begin{array}{l}\text { Paclitaxal + } \\
\text { B. diffusa }(100 \mathrm{mg} / \mathrm{kg})\end{array}$ & $6.05 \pm 0.53$ & $12.72 \pm 1.063$ & $\begin{array}{l}19.67 \pm 2.044 \\
* *\end{array}$ & $\begin{array}{l}26.83 \pm 2.834 \\
* * *\end{array}$ & $\begin{array}{l}35.72 \pm 3.172 \\
* * *\end{array}$ \\
\hline $\begin{array}{l}\text { Paclitaxel }+ \\
\text { B. diffusa }(200 \mathrm{mg} / \mathrm{kg})\end{array}$ & $6.05 \pm 0.45$ & $\begin{array}{l}10.44 \pm 1.004 \\
* * *\end{array}$ & $\begin{array}{l}17.22 \pm 1.109 \\
* * *\end{array}$ & $\begin{array}{l}21.28 \pm 2.081 \\
* * *\end{array}$ & $\begin{array}{l}27.33 \pm 2.271 \\
* * *\end{array}$ \\
\hline $\begin{array}{l}\text { Paclitaxel + } \\
\text { B. diffusa }(400 \mathrm{mg} / \mathrm{kg})\end{array}$ & $5.88 \pm 0.45$ & $\begin{array}{l}9.00 \pm 0.843 \\
* * *\end{array}$ & $\begin{array}{l}13.94 \pm 1.597 \\
* * *\end{array}$ & $\begin{array}{l}16.78 \pm 2.810 \\
* * *\end{array}$ & $\begin{array}{l}17.94 \pm 2.776 \\
* * *\end{array}$ \\
\hline
\end{tabular}

$* \mathrm{P}<0.05,{ }^{*} * \mathrm{P}<0.01, * * * \mathrm{P}<0.001$ compared with disease group.

Table-V: Effects of B. diffusa L., on locomotor activity in paclitaxel induced rats

\begin{tabular}{|c|c|c|c|c|c|}
\hline \multirow[t]{2}{*}{ Treatment groups } & \multicolumn{5}{|c|}{ Reaction time (Sec) $(n=6)$} \\
\hline & Day-0 & Day-7 & Day-14 & Day-21 & Day-28 \\
\hline Normal control & $113.5 \pm 6.53$ & $114.83 \pm 6.940$ & $116.00 \pm 6.293$ & $116.33 \pm 4.926$ & $117.50 \pm 5.822$ \\
\hline Paclitaxel (Disease control) & $112.83 \pm 6.5$ & $42.67 \pm 7.033$ & $38.83 \pm 6.494$ & $35.67 \pm 6.154$ & $39.33 \pm 7.005$ \\
\hline $\begin{array}{l}\text { Paclitaxel + Pregabalin } \\
(10 \mathrm{mg} / \mathrm{kg})\end{array}$ & $113.5 \pm 6.44$ & $60.67 \pm 4.227$ & $74.17 \pm 5.345$ & $83.50 \pm 5.753$ & $91.17 \pm 5.947$ \\
\hline $\begin{array}{l}\text { Paclitaxel + } \\
\text { B. diffusa }(100 \mathrm{mg} / \mathrm{kg})\end{array}$ & $114.83 \pm 6.49$ & $45.33 \pm 4.320$ & $48.50 \pm 4.506$ & $\begin{array}{l}52.33 \pm 5.086 \\
* * *\end{array}$ & $\begin{array}{l}55.83 \pm 4.53 \\
* * *\end{array}$ \\
\hline $\begin{array}{l}\text { Paclitaxel + } \\
\text { B. diffusa }(200 \mathrm{mg} / \mathrm{kg})\end{array}$ & $112.66 \pm 5.78$ & $50.17 \pm 5.636$ & $\begin{array}{l}56.17 \pm 6.616 \\
* * *\end{array}$ & $\begin{array}{l}64.67 \pm 6.772 \\
* * *\end{array}$ & $\begin{array}{l}69.67 \pm 6.21 \\
* * *\end{array}$ \\
\hline $\begin{array}{l}\text { Paclitaxel + } \\
\text { B. diffusa }(400 \mathrm{mg} / \mathrm{kg})\end{array}$ & $115 \pm 5.40$ & $\begin{array}{l}54.33 \pm 5.538 \\
*\end{array}$ & $\begin{array}{l}63.83 \pm 6.463 \\
* * *\end{array}$ & $\begin{array}{l}71.50 \pm 7.007 \\
* * *\end{array}$ & $\begin{array}{l}78.33 \pm 7.55 \\
* * *\end{array}$ \\
\hline
\end{tabular}

$* \mathrm{P}<0.05, * * \mathrm{P}<0.01, * * * \mathrm{P}<0.001$ compared with disease group. 
Table-VI: Effect of B. diffusa on Lipid peroxidation levels (TBARS), total protein, total calcium, superoxide dismutase levels ( $\mathrm{nM} / \mathrm{gm}$ of wet tissue) in sciatic nerve:

\begin{tabular}{|l|c|c|c|c|}
\hline Treatment groups & TBARS & Total Protein & Total Calcium & $\begin{array}{c}\text { Super Oxide } \\
\text { Dismutase (SOD) }\end{array}$ \\
\hline Normal control & $17.61 \pm 4.906$ & $74.08 \pm 8.181$ & $8.54 \pm 1.324$ & $45.09 \pm 7.080$ \\
\hline Paclitaxel (Disease control) & $54.55 \pm 10.296$ & $34.16 \pm 4.633$ & $26.7 \pm 2.654$ & $13.15 \pm 6.696$ \\
\hline $\begin{array}{l}\text { Paclitaxal+Pregabalin (10 } \\
\text { mg/kg) }\end{array}$ & $28.72 \pm 6.024$ & $64.16 \pm 3.204$ & $14.83 \pm 2.676$ & $35.50 \pm 4.399$ \\
\hline $\begin{array}{l}\text { Paclitaxel+ B. diffusa (100 } \\
\text { Mg/Kg) }\end{array}$ & $47.05 \pm 6.965$ & $39.83 \pm 4.854$ & $22.93 \pm 2.068$ & $22.37 \pm 3.328$ \\
\hline $\begin{array}{l}\text { Paclitaxel+ B. diffusa 200 } \\
\text { Mg/Kg }\end{array}$ & $42.05 \pm 7.864$ & $44.91 \pm 5.453$ & $\begin{array}{c}21.20 \pm 1.930 \\
* * *\end{array}$ & $23.33 \pm 4.190$ \\
\hline $\begin{array}{l}\text { Paclitaxel+ B. diffusa 400 } \\
\mathrm{Mg} / \mathrm{Kg}\end{array}$ & $\begin{array}{c}36.50 \pm 6.992 \\
* * *\end{array}$ & $\begin{array}{c}53.91 \pm 3.679 \\
* * *\end{array}$ & $\begin{array}{c}18.18 \pm 1.772 \\
* * *\end{array}$ & $\begin{array}{c}28.05 \pm 6.105 \\
* * *\end{array}$ \\
\hline
\end{tabular}

$* \mathrm{P}<0.05, * * \mathrm{P}<0.01, * * * \mathrm{P}<0.001$ compared with disease group.

\section{Discussion}

The purpose of present study is to examine the effectiveness of B. diffusa L., against Paclitaxel induced neuropathic pain assessed in terms of behaviour parameters, oxidative stress markers in sciatic nerve during 28 day study period. The results indicate the $\mathrm{B}$. diffusa $\mathrm{L}$., effectively inhibits Paclitaxel induced neuropathic pain from the day-7.

In the present study, by administering Paclitaxel (2 $\mathrm{mg} / \mathrm{kg}$, i.p) for 4 days leads to significant development of allodynia, hyperalgesia was noted in rodents from day-7. Similar findings are reported from previous studies Mangaiarkkarasi 19 who used Paclitaxel as neuropathic pain inducing agent. Paclitaxel always affects somatosensory system rather than motor system, ${ }^{20}$ where as vincristine disturbs both motor and sensory functions. ${ }^{21}$

Paclitaxel influence calcium dysregulation from swollen mitochondria ${ }^{22}$ involved in activation of calpin and caspases results in neuronal excitation. ${ }^{23}$ B. diffusa has well documented for limiting calcium conduction in nerve tissue, this could be due to the presence of liriodendrin has proven for its calcium limiting property and anticonvulsant property proven by. ${ }^{24,25}$

In addition Paclitaxel induced neuropathy decreases locomotor activity, similar findings were reported by Ray and Trammel ${ }^{26}$ during their four week study with Paclitaxel for assessing locomotor activity, sleep time, fatigue nature in rodents. The relation between sleep and neuropathic pain is bidirectional and may develop stress and sleep disorders. ${ }^{27}$ Stress intern raises corticosterone levels. ${ }^{28}$ However, rats treated with higher doses of B. diffusa L., were shown improvement in locomotor activity from day-14. Similar findings are reported with Meera sumanth and Mustafa ${ }^{29}$ in their antistress study by taking B. diffusa.

Further, Paclitaxel has been reported to cause neuropathic pain by producing reactive oxygen species. ${ }^{30}$ These reactive oxygen species involve in raising of lipid peroxidation levels in sciatic nerve. ${ }^{31}$ Paclitaxel potentially inhibit anti oxidant such as superoxide dismutase, will produce increased levels of superoxide anions and other reactive intermediates involve in generation of neuropathic pain. ${ }^{32}$ B. diffusa effectively improves the super oxide dismutase levels, and reduces lipid peroxidation levels due to its anti oxidant property. $^{33,34}$

On the basis of data in our hand and with support from the literature, it may be proposed that B. diffusa significantly attenuates the neuropathic pain induced by Paclitaxel. This could be due to its multiple effects; anticonvulsant, antioxidant, antistress, anti-inflammatory properties.

\section{Conclusion}

The present research open gates for the management of neuropathic pain with $B$. diffusa root extract due to its vast phytochemical properties. 


\section{References}

1. Campbell J. Mechanisms of neuropathic pain. Neuron, 2006, 52 (1) : 77-92.

2. Horowitz SH. The diagnostic workup of patients with neuropathic pain. Medical Clinics of North America, 2007, 91(1) : 21-30.

3. Jensen TS. Translation of symptoms and signs into mechanisms in neuropathic pain. Pain, 2003, 102(1-2) : 1-8.

4. Werhagen L, Budh CN, Hultling C, Molander C. Neuropathic pain after traumatic spinal cord injury relations to gender, spinal level, completeness, and age at the time of injury. Spinal Cord, 2004, 42 : 665-673.

5. Kanavos P. The rising burden of cancer in the developing world. Annals of Oncology, 2006, 17: 15-23.

6. Paice JA. Chronic treatment -related pain in cancer survivors. Pain, 2011, 152 : S8489.

7. Deng L, Guindon J, Vemuri KV, Thakur GA, White FA, Makriyannis ,Hohmann AG. The maintenance of cisplatin- and paclitaxelinduced mechanical and cold allodyniais suppressed by cannabinoid $\mathrm{CB} 2$ receptor activation and independent of CXCR4 signaling in models of chemotherapy-induced peripheral neuropathy, Mol Pain, 2012, $8: 71$

8. Driessen CM, Kleine-Bolt KM, Vingerhoets AJ, Mols F, Vreugdenhil G. Assesssing the impact of chemotherapyinduced peripheral neuro-toxicity on the quality of life of cancer patients. Support Care Cancer, 2012, 20(4) : 877-81.

9. Charity D. Scripture, William D. Figg and Alex Sparreboom. Peripheral Neuropathy Induced by Paclitaxel: Recent Insights and Future Perspectives. Current Neuropharmacology, 2006, 4 : 165-172.

10. Rowinsky EK, Eisenhauer EA, Chaudhry V,Arbuck SG, Donehower RC.Clinical toxicities encountered with paclitaxel
(Taxol), Semin Oncol, 1993, 20(4 Suppl 3) : 1-15.

11. Hilkens, P.H. \& ven den Bent, M.J. Chemotherapy-induced peripheral neuropathy. Journal of the peripheral nervous system, 1997, 2(4) : 350-361.

12. Cata JP, Weng HR, Lee BN, Reuben JM, Dougherty PM: Clinical and experimental findings in humans and animals with chemotherapy-induced peripheral neuropathy. Minerva Anestesiol, 2006, 72 : 151-169.

13. Arunachalam $M$ and Nirmal S. Attenuating effect of Acorus calamus extract in chronic constriction injury induced neuropathic pain in rats: an evidence of anti-oxidative, anti-inflammatory, neuroprotective and calcium inhibitory effects. BMC Complementary and Alternative Medicine, 2011, 11(1) : 24

14. Debjit B, Sampath Kumar K.P, Shweta S, Shravan P, Amit S, Dutta D. Traditional Indian Herbs Punarnava and Its Medicinal Importance. Journal of Pharmacognosy and Phytochemistry, 2012, 1(1) : 52-57.

15. Ujowundu CO, Igwe CU, Enemor VHA, Nwaogu LA, Okafor OE. Nutritive and Anti-Nutritive Properties of Boerhavia diffusa and Commelina nudiflora Leaves, Pakistan Journal of Nutrition, 2008, 7(1) : 90-92.

16. Sukhdev S, Suman Preet SK, Gennaro L, Dev Dutt R. Extraction technologies for medicinal and aromatic plants, International Centre for science and high technology, 2008.

17. Raghavan PV. Expert consultant CPSCEA, OECD guideline line. 2000.p. 420

18. sandeep kumar K, jameela Tahashildar, Karunakar Kota. Neuroprotective effect of ethanolic root extract of Boerhaavia diffusa (Linn.) against Streptozotocin induced Diabetic neuropathy in animal model. Journal of Chemical and 
Pharmaceutical Research, 2016, 8(3):831840.

19. Mangaiarkkarasi A, Rameshkannan S, Meher ali R. Effect of gabapentin and pregabalin in rat model of taxol induced neuropathic pain. Journal of clinical and diagnostic research, 2015, 9(5) : 11-14.

20. Xiao W.H, Zheng H, Zheng F.Y, Nuydens R, Meert T.F, Bennett G.J. Mitochondrial abnormality in sensory, but not motor, axons in paclitaxel-evoked painful peripheral neuropathy in the rat, Neuroscience, 2011, 29 : 461-469.

21. Postma T.J, Benard B.A, Huijgens P.C, Ossenkoppele G.J, Heimans J.J. Longterm effects of vincristine on the peripheral nervous system, Neurooncology, 1993, 15 : 23-27.

22. Flatters SJ, Bennet GJ. Studies of peripheral sensory nerves in Paclitaxel induced painful peripheral neuropathy: Evidence for mitochondrial dysfunction, Pain, 2006, 122 : 245-57.

23. Joseph EK, Levine JD. Caspase siganiling in neuropathic and inflammatory pain in the rat, European Journal of Neuroscience, 2004, 20 : 2896-2902.

24. Mandeep Kaur, Goel RK. Anti-Convulsant Activity of Boerhaavia diffusa: Plausible Role of Calcium Channel Antagonism. Evidence-Based Complementary and Alternative Medicine, 2011, 4 : 1-7.

25. Lami NS, Kadota S, Tezuka Y, Kikuchi T. "Constituents of the roots of Boerhaavia diff usa L. II. Structure and stereochemistry of a new rotenoid, boeravinone C2," Chemical and Pharmaceutical Bulletin, 1990, 38(6): 1558-1562.

26. Ray MA, Trammell RA, Verhulst S, Ran $S$, Toth LA. Development of mouse model for assessing fatigue during chemotherapy, Comparitive Medicine, 2011, 61(2) : 119130.
27. Cheatle MD, Foster S, Pinkett A, Lesneski $\mathrm{M}, \mathrm{Qu} \mathrm{D}$, Dhingra L. Assessing and managing sleep disturbance in patients with chronic pain, Anesthesiol Clin, 2016, 34(2) : 379-93.

28. Lira F.S. et al., Sleep pattern and locomotor activity are impaired by doxorubicin in non tumor-bearing rats, Sleep Science, 2016, 9 : 232-235.

29. Meera sumanth and Mustafa S.S. Antistress, Adoptogenic and Immunopotentiating Activity Roots of Boerhaavia diffusa in Mice. International Journal of Pharmacology, 2007, 3(5) : 416-420.

30. Fidanboylu M, Griffiths LA, Flatters SJ. Global inhibition of reactive oxygen species (ROS) inhibits paclitaxel-induced painful peripheral neuropathy, PLoS One, 2011, 6(9) : e 25212.

31. Feillet-Coudray C, Rock E, Coudray C, Grzelkowska K, Azais-Braesco V, Dardevet D, et al. Lipid peroxidation and antioxidant status in experimental diabetes, Clin Chim Acta, 1999, 284 : 31-43.

32. Hadzic T, Aykin-Bur ns N, Zhu Y, Coleman MC, Leick K, Jacobson GM, et al. Paclitaxel combined with inhibitors of glucose and hydroperoxide metabolism enhances breast cancer cell killing via $\mathrm{H} 2 \mathrm{O} 2$-mediated oxidative stress, Free Radic Biol Med, 2010, 48(8) : 1024-33.

33. Gopal TK, Harish G, Chamundeeswari D, Umamaheswara RC. In-vitro AntiOxidant Activity of Roots of Boerhaavia diffusa Linn. Research Journal of Pharmaceutical, Biological and Chemical Sciences. RJPBCS, 2010, 1(4) : 7828.

34. Aviello G, Canadanovic-Brunet JM, Milic N, Capasso R, Fattorusso E, Scafati TO, et al. Potent antioxidant and genoprotective effects of boeravinone $G$, a rotenoid isolated from Boerhaavia diffusa, PLos ONE, 2011, 6(5) : 19628. 